\title{
BMJ Open American and Australian family experiences while receiving a diagnosis or having treatment for idiopathic toe walking: a qualitative study
}

\author{
Cylie Williams (D) , ${ }^{1,2}$ Kristy Robson, ${ }^{3}$ Verity Pacey, ${ }^{4}$ Kelly Gray ${ }^{4}$
}

To cite: Williams C, Robson $\mathrm{K}$, Pacey V, et al. American and Australian family experiences while receiving a diagnosis or having treatment for idiopathic toe walking: a qualitative study. BMJ Open 2020;10:e035965. doi:10.1136/ bmjopen-2019-035965

- Prepublication history for this paper is available online. To view these files, please visit the journal online (http://dx.doi. org/10.1136/bmjopen-2019035965).

Received 28 November 2019

Revised 25 July 2020

Accepted 28 July 2020
Check for updates

(C) Author(s) (or their employer(s)) 2020. Re-use permitted under CC BY-NC. No commercial re-use. See rights and permissions. Published by BMJ.

${ }^{1}$ Allied Health, Peninsula Health, Frankston, Victoria, Australia ${ }^{2}$ School of Primary and Allied Health Care, Monash University, Frankston, Victoria, Australia ${ }^{3}$ School of Community Health, Charles Sturt University, Albury, New South Wales, Australia

${ }^{4}$ Department of Health

Professions, Macquarie University, Sydney, New South Wales, Australia

Correspondence to A/Prof Cylie Williams; cylie.williams@monash.edu

\section{ABSTRACT}

Objective To understand parent journeys while navigating diagnosis, assessment or treatment of their children with idiopathic toe walking (ITW).

Design Mixed methods qualitative study: analyses of survey data from the measure of processes of care-20 (MPOC-20) and semistructured interviews were analysed with an interpretative phenomenological analysis approach. Trustworthiness of data was achieved through member checking, researcher triangulation, reflexivity and transferability and comparison with the MPOC-20 results. Setting USA and Australia.

Participants Parents of children diagnosed with ITW who had seen more than one health professional during their care and lived in Australia or the USA.

Results Ten parents of children aged between 3 and 13 years and diagnosed with ITW participated. Parents described complex themes relating to their journeys. The themes relating to their journeys were: (1) riding the rollercoaster of diagnosis; (2) navigating the treatment options and (3) supporting parents in the journey. Each theme was supported by parent quotes about their experiences. Challenges were not localised to one country, in spite of vastly different healthcare systems.

Conclusions These findings create opportunities for an international approach to education, treatment recommendations and outcome measures to improve patient and parent experiences. Health professionals should consider the impact on parents in navigating between health professionals when provided with a diagnosis which can have variable outcomes and varied treatment options.

\section{INTRODUCTION}

Idiopathic toe walking (ITW) is a common childhood condition which can present in up to $5 \%$ of healthy, 5 -year-old children. ${ }^{1}$ ITW is diagnosed when a child over the age of 3 continues to walk with an absent or limited heel strike, and no other medical conditions known to cause toe walking. ${ }^{2}$ Some children have presented with challenges in sensory processing or singular motor development challenges. ${ }^{3}$ There is no single intervention used to treat ITW with documented full

\section{Strengths and limitations of this study}

- This study used family described experiences to understand the impact of care given to children who have idiopathic toe walking.

- Another strength was that these experiences were described in conjunction with a validated process of care measure.

- A limitation was that this study only included families who had seen more than one health professional during the course of their care.

- Another limitation may be self-selection bias, where families participated to tell their story of complex treatment journeys.

success in long-term gait normalisation. ${ }^{4}$ Treatments commonly aim at reducing associated ankle equinus, such as serial casting of the lower $1 \mathrm{leg}^{5}$ or surgical lengthening of the Achilles tendon. ${ }^{6}$ Other treatments, aim at physically restricting the toe walking gait, including ankle foot orthoses or full length foot orthoses. ${ }^{78}$ Reassurance or watchful waiting may be appropriate for some children, ${ }^{9}$ however, there is mounting evidence that treatment may be warranted. Sustained treatment effects have been demonstrated when the original concern was ankle equinus, ${ }^{10}$ despite studies reporting longterm toe walking post-treatment. ${ }^{610}$

A lack of robust ITW care pathways makes care difficult for healthcare providers, but even more so, parents. A recent survey of treatment options for ITW found that healthcare professionals preferred up to 10 different treatments and these varied depending on the healthcare professional. ${ }^{11}$ For parents, varied treatment options may result in a misinterpretation of an ITW diagnosis, its natural history or why their child received a different treatment to those offered to other children with the same diagnosis. 
Healthcare providers who develop a family-centred service focused on shared goals and relationships are commonly viewed more favourably by parents and children, even when the outcome may be unfavourable. ${ }^{12}$ Families with condition-specific knowledge and an understanding of the expected outcomes of treatment are commonly more engaged in shared treatment goals. ${ }^{13}$ Furthermore, in other conditions with ongoing care, engaged families are more likely to commit to prescribed home programmes, or adhere to gait aid recommendations. ${ }^{13-15}$

\section{Objectives of this study}

To identify and describe the parent journey while undergoing diagnosis, assessment or treatment of their child with ITW.

\section{METHODS}

A mixed methods research design was chosen to explore the understanding of family experiences while children are undergoing assessments and treatment for ITW. The consolidated criteria for reporting qualitative research checklist was used to guide reporting. ${ }^{16}$ Using a qualitative approach enabled the focus to be on the participants' understanding related to the experience and a questionnaire increased the robustness of the data. ${ }^{17}$ Specific to this study, the approach taken was interpretative phenomenological analysis (IPA) due to its attention towards the meaning of everyday life experiences and the individual's interpretation of these experiences. ${ }^{18}$ Key to IPA is data collection and analysis from a small number of purposively selected participants who have a mutual experience, enabling a detailed analysis of phenomena by incorporating each participant's unique experience through an idiographic process. ${ }^{19}$

\section{Participant recruitment}

We applied a purposive sampling approach to recruitment. Eligible parents were recruited if they lived in Australia or the USA, had a child under the age of 18 , who had been diagnosed with ITW by a medical health professional, seen at least two healthcare professionals through their child's course of care, and had received treatment for the ITW within the past 12 months. We recruited from Australia and the USA as we were interested in understanding if there were differences in experiences between diverse healthcare systems. Advertisments inviting parents who met the inclusion criteria were placed on social media (Facebook or Twitter), both on toe walking support group pages and health professional pages (eg, Monash University Physiotherapy). Parent responses were screened through first emailed questions about the history of who diagnosed their child with ITW and if there were any other health or medical problems present known to cause toe walking gait. This was also confirmed verbally during the interview. These questions were based on part of a exclusionary tool for diagnosis of ITW. ${ }^{2}$

\section{Data collection}

Following consent, parents were provided with an online link to anonymously complete the measure of processes of care-20 (MPOC-20). The MPOC-20 contains 20 questions over five domains: Enabling and partnership, Providing general information, Providing specific information, Coordinated and comprehensive care, Respectful and supportive care. The MPOC-20 is scored on a seven-point Likert scale response from 1 (not at all) to 7 (to a very great extent). Its psychometric properties have been tested, with excellent internal consistency (factor alphas ranging between 0.83 and 0.92 ), retest reliability (interclass correlation coefficient ranging between 0.81 and 0.86 ) and construct and concurrent validity. ${ }^{20}$ Single individual semistructured online recorded video interviews were undertaken with parent participants by a single researcher (CW) who is a health professional and had extensive experience in assessing, treating and researching children who have ITW. To ensure that data collection and analysis was undertaken with a degree of open-mindness, bracketing principles of IPA were employed. ${ }^{19}$ These included interview questions developed by CW, KG, VP, who have diverse paediatric clinical experience, two parents who have lived experience, and KR who has extensive qualitative research experience. Questions were designed to ensure no leading guidance to participants or direction for responses. Participant interviews were guided by openended question approaches such as 'Can you discuss how well you initially understood this diagnosis?' Questions were phrased in a manner to encourage discussion and where needed, additional probing questions were used, such as 'what advice were you given about the diagnosis?'. Each participant was guided through a semistructured interview to talk about their their experiences through understanding the ITW diagnosis, how treatments were offered, what types of treatments had been explored, understanding outcomes or treatment success and future suggestions for health professionals and other parents (online supplementary file).

Interviews were recorded by the first researcher (CW), transcribed verbatim (by a third party transcription service), and emailed to participants for member checking and feedback prior to commencement of data analysis.

\section{Data anaylsis}

Within an IPA study, the sample size is based on the richness of data and analysis depth from the individuals participants, as well as the design of the study. ${ }^{19}{ }^{21}$ Interviews were undertaken with one or both parents therefore grouped participant data were collalated, together with information about their child who was diagnosed with ITW. The MPOC-20 data scored as per guidelines. ${ }^{22}$ Participants ranked statements from $1=$ not at all to $7=$ to a very great extent. The scores were averaged to form an 
individual domain score. Group domain scores were summarised in medians, IQRs and ranges, where the higher the score, the greater agreement or engagement was ranked in that domain. To avoid preconceptions, qualitative data were initially analysed by a single researcher (KR), who has limited prior clinical and research experience in the specific topic area of ITW, using the IPA guideline as described by Smith et al. ${ }^{19}$ As part of this process, themes were independently developed and drafted by the second researcher (KR) and further reviewed by the first researcher $(\mathrm{CW})$.

Data analysis was undertaken through a four-step process:

1. Individual transcripts were read multiple times, enabling familiarity of individual stories.

2. Descriptive notes and initial nodes were made using NVivo V.11 software, to highlight key points.

3. Nodes were then used to identify evolving themes unique to each participant's transcript, with similar themes being grouped together.

4. Following analysis of individual transcripts, emerging themes were compared and contrasted between individual transcripts to create overarching super-ordinate themes.

Consideration was undertaken to identify any distinct differences between participants residing in either country. No discernible geographic differences were noted that would influence the super-ordinate themes. Therefore, no separate participants per country analysis was undertaken.

Trustworthiness of data was achieved through member checking, researcher triangulation, reflexivity and transferability and comparison with the MPOC-20 results. ${ }^{23}$ Following transcription, the interviewer (CW) reviewed the transcript to ensure it was reflective of the interview questions asked, then through the member checking process. ${ }^{23}$ To limit the risk of individual bias, researcher triangulation was undertaken by involving two of the authors in the data analysis phase. ${ }^{24}$ This was by themes independently developed and drafted by the second researcher (KR) and further reviewed by the first researcher (CW) to ensure authenticity with the lived experience of the participants. Acknowledgement of researcher's personal bias and the potential influence on research findings is important to address through a process of reflexivity. ${ }^{25}$ The second researcher (KR) independently reviewed the video recording of each interview and kept a reflective journal to document thoughts and insights from the participant stories, including both verbal and non-verbal elements. During the data analysis phase, the researcher (KR) continued to use the reflective journal to document and build on understandings, thereby facilitating an opportunity to add depth to the research findings. ${ }^{26}$ In addition, a detailed description of the participants was provided to further enable judgements of transferability of the study. ${ }^{23}$

\section{Patient and public involvement}

Two parents with lived experience of having a child diagnosed and treated for ITW provided input into the question design.

\section{RESULTS}

Interviews were conducted between May and November 2018 and each interview ranged between 35 and $60 \mathrm{~min}$. There were 21 parents who responded to take part in the research, subsequent to screening questions, there were 13 who consented to participate with 10 completing the interview. The three who did not participate was due to either child illness or difficulty in scheduling a time with the researcher. Table 1 presents the participant pseudonyms, gender of child diagnosed with ITW, country of residence and list of health professionals who had previously or currently been involved in their child's care for ITW. Table 2 provides the MPOC-20 scores for each of the domains. The median domain scores were used during data checking.

Three super-ordinate themes emerged through the analysis of the interviews with parents of children who had been diagnosed with ITW. These were: (1) riding the rollercoaster of diagnosis; (2) navigating the treatment options and (3) supporting parents in the journey.

\section{Theme 1: Riding the rollercoaster of diagnosis}

Participants described two subordinate themes within this theme leading to the analogy of riding the highs and lows of a rollercoaster. These subthemes included: (a) Varied clinical knowledge of how ITW presents and is treated and, (b) breaking down the complexity of the ITW diagnosis.

Difficulty in obtaining a diagnosis was described as a challenged faced by parents due to the Varied clinical knowledge of how ITW presents and is treated by health professionals. This challenge resulted in numerous appointment reviews of children by multiple health professionals during this rollercoaster of diagnosis. All participants spoke about conflicting information provided by health professionals during the process of diagnosing their child with ITW. This confusion and disparity in information provision was reflected through the MPOC-20 scores where the domain Providing general information was rated the lowest of all domains. Some participants described their dissatisfaction in health professionals dismissing their concerns about their child while a diagnosis is being explored. Jasmine highlights this point when talking about her diagnosis experience.

So she was diagnosed by a paediatric physio and I had her assessed twoyears prior to that and the podiatrist said no, she wasn't a toe walker, that there was nothing wrong with her. So I initially was told nothing wrong, don't worry about it, she is going to grow out of it. So left it for a while, then thought no, this isn't right. So ended up with a paediatric physio who diagnosed her. (Jasmine) 


\begin{tabular}{|c|c|c|c|}
\hline Pseudonym & Country & Age of child with ITW & Health professionals involved with diagnosis and treatment of ITW \\
\hline Kelly & USA & 5 years, female & $\begin{array}{l}\text { Primary care doctor, physical therapist, orthopaedic surgeon, } \\
\text { psychologist, neurologist, geneticist }\end{array}$ \\
\hline Tara & USA & 5 years, female & $\begin{array}{l}\text { Occupational therapist, physical therapist, paediatrician, physiatrist, } \\
\text { orthopaedic surgeon }\end{array}$ \\
\hline Krista & Australia & 8 years, female & Paediatrician, physiotherapist, orthotist, podiatrist \\
\hline Anna & USA & 13 years, female & $\begin{array}{l}\text { Paediatrician, physical therapy, podiatrist, orthopaedic surgeon, pain } \\
\text { clinic }\end{array}$ \\
\hline Melissa & Australia & 7 years, male & Paediatrician, rehabilitation physician, physiotherapist, podiatrist, \\
\hline Jasmine & Australia & 9 years, female & General medical practitioner, physiotherapists, podiatrist, chiropractor \\
\hline Robyn & Australia & 13 years, female & Paediatrician, physiotherapist, chiropractor, podiatrist \\
\hline Yvonne & USA & 12 years, female & $\begin{array}{l}\text { Paediatrician, physical therapist, unknown medical specialist, } \\
\text { orthopaedic surgeon, podiatrist }\end{array}$ \\
\hline Cindy & USA & 3 years, male & $\begin{array}{l}\text { Paediatrician, orthopaedic surgeon, orthotist, physical therapy, } \\
\text { chiropractor }\end{array}$ \\
\hline Sarah & USA & 5.5 years, male & Paediatrician, orthopaedic surgeon, physical therapist \\
\hline
\end{tabular}

ITW, idiopathic toe walking.

Participants commonly talked about the lack of general awareness of the condition which often resulted in a delay in obtaining a diagnosis as can be seen from Anna's excerpt.

She always walked tiptoed. I would say the first time I asked about it was probably about age four at her pediatrician. And at the time she still had full range of motion, so her pediatrician was not overly worried about it. She said, 'Well just keep an eye on it, and see how it goes'. It wasn't maybe until a couple of years later that I had it checked again ... I would say when she was nine is the first time that I really was like; 'You can't pull your foot up' meaning she doesn't have full range of motion anymore. (Anna)

Anna's experience demonstrates how difficulty obtaining a diagnosis may delay treatment which could potentially reduce any long-term impact.

The challenge for some parents when health professionals dismissed their concerns was also highlighted by many participants. They talked about their child's toe

\begin{tabular}{|c|c|c|}
\hline & $\begin{array}{l}\text { Median } \\
\text { (IQR) }\end{array}$ & Range \\
\hline Enabling and partnership & $5(4-6)$ & $3-7$ \\
\hline Providing general information & $2(2-3)$ & $1-6$ \\
\hline $\begin{array}{l}\text { Providing specific information } \\
\text { about the child }\end{array}$ & $5(4-6)$ & $2-7$ \\
\hline $\begin{array}{l}\text { Coordinated and } \\
\text { comprehensive care }\end{array}$ & $6(4-6)$ & $2-7$ \\
\hline Respectful and supportive care & $5(4-6)$ & $3-7$ \\
\hline
\end{tabular}

MPOC-20, measure of processes of care-20. walking being described as a habit, and something they would grow out of it. Sarah explained:

Well, I understood it to be more of its possibly a habit, something that he would outgrow by school age, I think it was kind of explained to us. By the age of five to seven he could just outgrow it, the specialist suggested that physical therapy would maybe be a good route to just make sure that we were strengthening any areas that he had weaknesses in, but other than that he didn't see it as anything more than a habit, which is just how I thought of it. (Sarah)

Sarah's quote illustrates how parents may interpret information provided to them by health professionals. In some cases, a narrow viewpoint may be provided based on the health professionals experience in managing this condition. This was also echoed by Robyn who also explained how the initial interaction with her general practitioner ultimately meant that she delayed getting treatment for her child.

We kind of mentioned it to our GP [general practitioner], well I went to the GP with my daughter, I think for something else and mentioned it. And he said 'well look, she'll grow out of it', the GP kind of fobbed us off, and yeah not until the pain came did we kind of look for something else. (Robyn)

In the absence of health professionals providing more detailed information regarding a diagnosis of ITW, parents may delay accessing treatment.

Participants also talked the challenges associated with breaking down the complexity of the diagnosis, often having to balance their own fact finding on ITW with the information provided by their health professionals. Yvonne explained: 
I was reading about autism and all that type of stuff. Nobody ever wanted to get her tested for any kind of sensory-any sensory problems or testing her for autism, which-that was kind of strange, based on the facts that they couldn't find a reason. I'd like to find a reason, just to rule that out, but nobody's ever offered that. (Yvonne)

For Yvonne, her perception of not being supported by her child's health professionals to rule out all underlying causes, meant it was difficult for her to understand why it was occurring, and to know how best to help her child. However, not all participants had this experience as can be seen from Melissa's excerpt.

The podiatrist is really good as he puts it into every day speak for me and gets rid of all the gobblygook. But that suited me fine because, you know, if I had to read any sort of articles, or any sort of literature on it, I'd probably, you know, wouldn't understand the scientific terminology as much as they do so I was quite happy for them to make up what it meant. (Melissa)

Melissa highlighted the importance of ensuring information provided to parents about the diagnosis and management for ITW is in a form that easily understood by parents and enables them to be actively part of the decision-making process for their child.

\section{Theme 2: Navigating the treatment options}

The MPOC-20 domain Coordinated and comprehensive care was one of the higher rated domains by participants, however, during the interviews participants talked about being provided with limited information about the range of treatment options for ITW management. This lead to a number of subthemes throughout the navigation of treatment options. These included (a) Let's do it my way; (b) Establishing a clear treatment pathway and (c) Managing the practicality of treatment options.

Participant's described a lack of understanding on the types of treatments available, rather a let's do it my way approach was commonly applied when exploring treatment options. This can been seen from Jasmine's statement.

The paediatric physio was not at all interested in trying anything else other than the casting. He was adamant that we were wasting our time and that he would not do anything other than casting. He just completely ruled out any other option. So yeah, with him, 'If you see me this is what you are doing. If you are not doing this well, then you are not seeing me.' (Jasmine)

Jasmine's experience highlights the challenge some parents may face when understanding the range of options available if their health professional has a particular belief on only one approach.

Participants also spoke about the difficulty in deciding which management pathway to choose for their child, in the absence of having access to clear evidence-based information. Participants appeared to understand there was no standardised approach to managing ITW, but rather this was dependent on the preferences of the individual health professional. Tara explained:

I think each one you could feel their personal biases a little bit. You know, the orthopedist for sure is recommending surgery, physiatry (rehabilitation specialist) was for sure going to be a botox and casting, therapists are like let's work with the orthotists and make braces. But the therapists, I think, you know, pretty much were the most honest in that in saying let's do exercises, let's do bracing, let's do these things so that you can pick when you want to do the surgery. (Tara)

Tara's quote illustrates the difficulty that parents can face when every health professional has a different viewpoint, or introduces a treatment plan that has staggered interventions with the parent not understanding the context of this treatment approach.

While it was acknowledged by participants that the management of their child's ITW was often not straightforward, this was complicated by a limited focus on establishing a clear treatment pathway. This resulted in some parents not feeling reassured they were making the right decisions. It was particularly evident when their health professionals were using a trial and error approach without much discussion on a long-term plan. Kelly highlighted this point:

The original doctor didn't explain to me anything about what was going on, what we would do. Having a plan at least, maybe a year out, like this is what we'll do at three months and six months, would make me feel reassured that the doctor knew what he was doing, and that I have control over what's going on. Compared to not knowing what's happening next. (Kelly)

A lack of certainty about treatment success and treatment progression, especially when treatments were not working, meant that for some participants they felt a lack of control in being empowered to know they had made the right decision. However, other participants had a different experience with their healthcare providers. Krista talked about having a clear plan moving forward.

Her orthopedic is very open to discussing what she wants to do and why, and if I have an idea, she'll tell me whether we should wait or do it, or anything of that nature. (Krista)

For some participants, establishing a clear pathway was further complicated by access to health services. In Cindy's case she chose to try a conservative approach first, physical therapy, despite the surgeon warning her not to, as she would require the physical therapy visits after surgery. 
So, now we're into physical therapy. The physical therapy, I made the call on that because the surgeon is saying don't use your physical therapy days, because if we do surgery, you're going to need those. But, as a mum, I'm going we have to try physical therapy before we try surgery. So, I made the call on that and now he's in physical therapy that he just started I think two weeks ago to try and do something to come down. (Cindy)

When there is limited or poorly timed access to health services it may make it more difficult for parents to decide how to proceed. Or it may impact on the long-term prognosis for the child, as can be seen by Tara's experience.

I mean, by the time we already decided we were having the surgery, we went a month without AFOs because he had outgrown them and couldn't get a new pair and knowing we needed it, I think he lost 10 degrees (Tara)

Alongside the challenge of understanding and then deciding on treatment options, there was an also an appreciation by the participants of the complexity of ITW and the unique set of circumstances relevant to each child. Tara captured the feeling of other participants when she described her viewpoint.

You really see that it's much more complicated and each kid is very, very unique. That there are sensory pieces, there's the physical shortening of the heel cord, there is this whole process that his body posture has changed as a result of this, that it's much bigger than just he's walking on his toe, stick some braces on and in a year or so he'll stop. (Tara)

Participants recognised the need to have a holistic approach towards managing ITW, however, there was a perception that this was not always the approach taken by all health professionals managing their child with ITW.

In addition to the challenges of not having clear treatment pathways, participants noted frustrations when there was minimal consideration by some health professionals on managing the practicality of treatment options. This was also reflected in some of the scores within the MPOC20 domains Providing specific information for my child and Respectful and coordinated care. For some participants this related to having their child in plaster casts or in Robyn's experience where it centred on having to facilitate her child to do multiple exercises.

I've found it frustrating that there were so many different exercises, like one visit there' $d$ be this type and then the next visit leave that, you know that wasn't working we'll go to the next one and she was only 11-ish at the time, but um, you know really not an excuse but we're busy working, we've got two other children and just to get her to do everything was really tricky. And each week, you know each kind of visit it would change, and I think she found that really hard. (Robyn)
Robyn's experience demonstrates the obstacles some parents face when trying to juggle family life in addition to the treatment options implemented by health professionals. The emotional toll of undertaking treatments and not having success was difficult for both parent and child to deal with, especially when limited information had been provided on the potential outcomes or clear explanations on the likely success of treatments. For some participants, this ultimately made the decision on treatment pathways difficult to navigate.

\section{Theme 3: Supporting parents in the journey}

Participants commonly talked about not being supported to understand the journey of a child who is diagnosed ITW. These reflections were closely related to some of the lower scores within the MPOC-20 domain Enabling and partnership and again Providing general information. Subthemes of (a) I'll do it myself; (b) That didn't go as planned and (c) Being connected were identified as participants described their challenges over their long treatment journeys.

I'll do it myself was clearly described by many participants through their journey of treatment. Cindy explains how she had to rely on her own research to find out the answers that she needed.

I haven't gotten too much information from doctors. They kind of give you the diagnosis and they send you off for AFOs and you get those and then you are kind of on your own until you go back to see the doctor in six months. So, just having to do my own research and why are we putting him in AFOs now? Why is he doing physical therapy now? (Cindy)

Cindy's experience demonstrates that if some parents have to seek information from sources other than their health professionals, they may not always be getting the most accurate or evidence-based information.

Participants also talked about the sense of frustration that occurred when treatments were unsuccessful, resulting in a perception by parents of that didn't go as planned. For Anna, she felt that she was given false promises when agreeing for her child to undertake surgery.

I feel like when he talked about the surgery he acted like everything was going to be fixed. And that wasn't the case. (Anna)

A disconnect between treatment expectations and outcomes, especially with invasive treatment options, may have an emotional impact on both parent and child. This may be further exacerbated if health professionals do not have honest conversations with parents. Whereas for some participants they had learnt over their journey to have a more pragmatic approach to treatment success. Kelly explains:

I used to be very hopeful that the next doctor was going to have a solution for me and have her back on 
her heels. I've just learned and taken it one day at a time and we'll see what happens. (Kelly)

Kelly's excerpt highlights the challenge some parents may face when there is little support to enable parents to understand the condition. Alongside this, participants also talked about an element of guilt on whether they had done the right thing, should they have done something different. This is highlighted in Robyn's account.

And I, you know, I feel quite guilty that you know, got photos of her, you know, toe walking in a fairy dress thinking how cute, you know we just didn't even think anything of it. And we joke now, she blames us that she has this problem, but I worry for the long term, you know, physical implications, um because she is always in pain. (Robyn)

The worry of not knowing when the journey would end or what the long-term outcomes were going to be for their child was a common feeling among participants. Despite this, participants highlighted that being connected with support groups where parents could network and described their own lived experiences was useful. Kelly explained:

There's a support group on Facebook for toe walkers. There was just a lot of people talking positively about their experience with serial casting, so that's when my husband and I, we-the surgery thing, we saw the kids-pictures with the kids going through the surgeries and stuff. We just knew that wasn't for us. We knew-seeing the pictures of the other children's feet before and comparing it to my daughter's, where our daughter's was not anywhere as severe as the ones who had had the surgery. I was able to make our decision based on comparing severity to what I was seeing. That was very helpful. (Kelly)

Kelly spoke how support groups made it easier for her and her husband to make decisions on what treatment options they could consider. Being connected to other parents going through the same situation was helpful to participants in knowing what treatment options were available and what was working for other children.

Participants commonly recognised that ITW was a complex diagnosis which was unlikely to be rapidly fixed and that treatments which succeeded with one child may not work for another. This is illustrated in Melissa's excerpt.

So, sometimes you do have to go on that journey, as much as it's frustrating for you and them, and everyone involved, I think sometimes, I understand you have to go through those sorts of things so you know it's just finding the right treatment for the right child for what's happening. (Melissa)

Participants highlighted that parents should be made aware of the challenges when a diagnosis of ITW is made and the ability to be connected with others may assist in this appreciation.

Finally, all participants described their daily struggles associated with their individual circumstances and the need to advocate to get the best outcome for their child. Kelly encapsulated the opinion of other participants when she described her advice for other parents.

You need somebody who is sympathetic and understanding and willing to really work with the individual needs of the child, not one shoe fits all kind of thing. (Kelly)

Kelly illustrates the importance for parents to be empowered to actively seek out health professionals that are collaborative in their approach and are willing to support them throughout the journey.

\section{DISCUSSION}

This is the first study to explore international parent perceptions and challenges while navigating the diagnosis and treatment for their child diagnosed with ITW. It was surprising to find that these challenges were not localised to one country even with vastly different healthcare systems and referral pathways. Yet, these findings create many opportunities for an international approach to education, treatment recommendations and outcome measures to improve patient and parent experiences.

The themes identified within this research were similar to those commonly seen for parents who navigate the healthcare system with a child who has a complex and variably presenting health condition. ${ }^{13}$ These themes including the ups and downs during diagnosis, moving between multiple healthcare providers who had different opinions on care and recommendations to healthcare professionals on how they can support their journey better. These themes embody the notion of partnership and empowerment for better outcomes, similarly to those described by parents of children with cerebral palsy. ${ }^{13} 27$

Parents emphasised their need for healthcare providers to use consistent language, information and treatment about ITW. Common paediatric healthcare information and treatments are increasingly standardised globally due to an increased number of position papers or Cochrane reviews. These are regularly published for conditions such as paediatric flat foot ${ }^{28-30}$ or congenital talipes equinovarus ${ }^{31}$ yet there is only one known treatment protocol for ITW, published by the Physical Therapy Association of America ${ }^{32}$ amid a number of limited systematic reviews with no meta-analysis to guide interventions. ${ }^{433} 34$

There is also a call for consistency in valid outcome measures to understand treatment impact ${ }^{35}$ for this population group. The disparity observed in a recent systematic review highlights that in research, there are a variety of methods and tools for measurement of outcomes, of which parent reported measure rarely feature. This inherently will translate to challenges in clinical practice, where health professionals do not have a gait laboratory 
environment but need simple and cost-efficient measures to understand their treatment success. Ultimately though, treatment success and what success looks like should be a joint decision within the clinical setting. A recent Cochrane review found the only reported parent outcome measure was the percentage of time the child toe walked. This parent reported outcome was described in by one of the four included studies, ${ }^{4}$ yet in this present qualitative study, very few parents talked about consistent goal setting with their health professionals or this being an outcome of interest.

The participants provided a number of recommendations to increase knowledge of other parents who have a child diagnosed with ITW. They suggested easy-to-access evidence-based information resources, such as a websites or standardised handouts. These resources are usually provided by hospitals, foundations or international clinical reference groups. Participants highlighted having varied treatment options prescribed by different health professionals, demonstrating the challenge of an absence of standard care pathways. Similarly, described in a recent international health professional's survey. ${ }^{11}$ Without international uniformity of treatment pathways, parents and children may continue to receive disparate care. It should be a priority for healthcare providers and researchers to provide information consistent with evidence.

This research has a number of limitations that are inherent to qualitative studies. A key limitation is the self-selection bias of the participants. This interview was focused on the patient journey, rather than recruitment phrased in a negative light. However, this may have inherently attracted participants who had a difficult time through any of the stages of diagnosis and treatment. While the research team carefully considered the language of recruitment, this may impact on the generalisability to other parents who are navigating assessment and treatment. Despite reaching saturation of these, it is possible that this small sample size may have inadvertently over or understated the importance of the themes and their impact.

The strengths of this research are the depth and shared challenged of parents that health professionals may have never considered. This highlights focused areas for research and healthcare. The primary outcome should be the consideration of consistent language and outcomes antiscipated by health professionals in the education of parents during the diagnosis and treatment phase. It is unknown if a consistent template or treatment protocol would improve parent and patient experience or even if this is an issue for the children. Future research may consider the impact of the child through this process and if this is more of a burden for the parent, or the child is actually impacted through the treatment episodes.

\section{CONCLUSION}

ITW is thought to be a benign gait condition that many children grow out of, or have minor long-term effects.
However, it appears this is a condition that parents struggle to obtain consistent advice and treatment recommendations, while often advised to see multiple healthcare providers. There is a need for consistent education and knowledge about this condition to improve the outcomes or satisfaction with outcomes for the families of children with ITW.

\section{Twitter Cylie Williams @cyliepaedspod}

Acknowledgements Thank you to the parents who provided input into the questions, and those who participated in the research

Contributors CW conceived the study. CW, KR, VP and KG equally designed the study and the questions. CW undertook the data collection. KR and $\mathrm{CW}$ undertook the data analysis. CW, KR, VP and KG equally interpreted the data. CW, KR and KG drafted the manuscript draft and circulated to authors for contribution. CW, KR, VP and $K G$ approved the current manuscript version of the manuscript for publication.

Funding CMW is supported by a National Health and Medical Research Council Early Career Research Health Professional Fellowship.

Competing interests None declared.

Patient consent for publication Not required.

Ethics approval Monash University Human Research Ethics Committee, Victoria, Australia approved this study (MUHREC approval: 11717). All participants consented to participate prior to data collection.

Provenance and peer review Not commissioned; externally peer reviewed.

Data availability statement All data relevant to the study are included in the article or uploaded as supplementary information. Interview guide is provided within supplementary data.

Open access This is an open access article distributed in accordance with the Creative Commons Attribution Non Commercial (CC BY-NC 4.0) license, which permits others to distribute, remix, adapt, build upon this work non-commercially, and license their derivative works on different terms, provided the original work is properly cited, appropriate credit is given, any changes made indicated, and the use is non-commercial. See: http://creativecommons.org/licenses/by-nc/4.0/.

ORCID iD

Cylie Williams http://orcid.org/0000-0002-0223-9141

\section{REFERENCES}

1 Engström P, Tedroff K. The prevalence and course of idiopathic toewalking in 5-year-old children. Pediatrics 2012;130:279-84.

2 Williams CM, Tinley P, Curtin M. The toe walking tool: a novel method for assessing idiopathic toe walking children. Gait Posture 2010;32:508-11.

3 Williams CM, Tinley P, Curtin M, et al. Is idiopathic toe walking really idiopathic? the motor skills and sensory processing abilities associated with idiopathic toe walking gait. J Child Neurol 2014;29:71-8.

4 Caserta AJ, Pacey V, Fahey M, et al. Interventions for idiopathic toe walking. Cochrane Database Syst Rev 2019;10:CD012363.

5 Fox A, Deakin S, Pettigrew G, et al. Serial casting in the treatment of idiopathic toe-walkers and review of the literature. Acta Orthop Belg 2006;72:722-30.

6 Eastwood DM, Menelaus MB, Dickens DR, et al. Idiopathic toewalking: does treatment alter the natural history? J Pediatr Orthop B 2000;9:47-9.

7 Herrin K, Geil M. A comparison of orthoses in the treatment of idiopathic toe walking: a randomized controlled trial. Prosthet Orthot Int 2016;40:262-9.

8 Michalitsis J, Murphy AT, Rawicki B, et al. Full length foot orthoses have an immediate treatment effect and modify gait of children with idiopathic toe walking. Gait Posture 2019;68:227-31.

9 Stricker SJ, Angulo JC. Idiopathic toe walking: a comparison of treatment methods. J Pediatr Orthop 1998;18:289-93.

10 Davies K, Black A, Hunt M, et al. Long-Term gait outcomes following conservative management of idiopathic toe walking. Gait Posture 2018;62:214-9.

11 Williams CM, Gray K, Davies N, et al. Exploring health professionals' understanding of evidence-based treatment for idiopathic toe walking. Child Care Health Dev 2020;46:310-9. 
12 Carlsen B, Glenton C, Pope C. Thou shalt versus thou shalt not: a meta-synthesis of GPs' attitudes to clinical practice guidelines. $\mathrm{Br} \mathrm{J}$ Gen Pract 2007;57:971-8.

13 Rosenbaum P. Cerebral palsy: what parents and doctors want to know. BMJ 2003;326:970-4.

14 Kruijsen-Terpstra AJA, Verschuren O, Ketelaar M, et al. Parents experiences and needs regarding physical and occupational therapy for their young children with cerebral palsy. Res Dev Disabil 2016;5354:314-22.

15 Russell S, McCloskey CR. Parent perceptions of care received by children with an autism spectrum disorder. J Pediatr Nurs 2016;31:21-31.

16 Tong A, Sainsbury P, Craig J. Consolidated criteria for reporting qualitative research (COREQ): a 32-item checklist for interviews and focus groups. Int J Qual Health Care 2007;19:349-57.

17 Flick U. Designing qualitative research. London: Sage Publications Ltd, 2008.

18 Finlay L. Phenomenology for therapists: researching the lived world. United Kingdom: John Wiley \& Sons, 2011.

19 Smith J, Flowers P, Larkin M. Interpretative Phenomenological Analysis. In: Theory, method and research. London: Sage Publications Ltd, 2009.

20 King S, King G, Rosenbaum P. Evaluating health service delivery to children with chronic conditions and their families: development of a refined measure of processes of care (MPOC-20). Children's Health Care 2004;33:35-57.

21 Pietkiewicz I, Smith JA. A practical guide to using interpretative phenomenological analysis in qualitative research psychology. Czasopismo Psychologiczne 2014;20:7-14.

22 Saleh M, Almasri NA. Use of the measure of processes of care (MPOC-20) to evaluate health service delivery for children with cerebral palsy and their families in Jordan: validation of Arabictranslated version (AR-MPOC-20). Child Care Health Dev 2014;40:680-8.
23 Curtin M, Fossey E. Appraising the trustworthiness of qualitative studies: guidelines for occupational therapists. Aust Occup Ther $J$ 2007;54:88-94.

24 Denzin NK. Interpretive biography. Thousand oaks,CA: Sage, 1989.

25 Finlay L. The reflexive journey: Mapping multipleroutes. In: Gough B, ed. Reflexivity: a practical guide for researchers in health and social sciences. Oxford: Blackwell Science Ltd, 2003: 3-20.

26 Davis J, Watson N, Cunningham-Burley S. Learning the lives of disabled children: Developing a reflexive approach. In: Christensen P, James A, eds. Research with children: perspectives and practices. London: Falmer Press, 2000: 201-24.

27 Cooley WC, American Academy of Pediatrics Committee on Children With Disabilities. Providing a primary care medical home for children and youth with cerebral palsy. Pediatrics 2004;114:1106-13.

28 Dars S, Uden H, Banwell HA, et al. The effectiveness of non-surgical intervention (foot Orthoses) for paediatric flexible PEs planus: a systematic review: update. PLoS One 2018;13:e0193060.

29 Rome K, Ashford RL, Evans A. Non-surgical interventions for paediatric PEs planus. Cochrane Database Syst Rev2010;7.

30 Carr JB, Yang S, Lather LA. Pediatric PEs planus: a state-of-the-art review. Pediatrics 2016;137:e20151230.

31 Gray K, Pacey V, Gibbons P, et al. Interventions for congenital talipes equinovarus (clubfoot). Cochrane Database Syst Rev 2014;29.

32 Le Cras S, Bouck J, Brausch S, et al. Cincinnati Children's Hospital Medical Center: Evidence-Based Clinical Care Guideline for Management of Idiopathic Toe Walking, 2011.

33 van Kuijk AAA, Kosters R, Vugts M, et al. Treatment for idiopathic toe walking: a systematic review of the literature. J Rehabil Med 2014:46:945-57.

34 van Bemmel AF, van de Graaf VA, van den Bekerom MPJ, et al. Outcome after conservative and operative treatment of children with idiopathic toe walking: a systematic review of literature. Musculoskelet Surg 2014;98:87-93.

35 Caserta A, Morgan P, Williams C. Identifying methods for quantifying lower limb changes in children with idiopathic toe walking: a systematic review. Gait Posture 2019;67:181-6. 